CLAWAR 2018: 21st International Conference on Climbing and Walking Robots and the Support Technologies for Mobile Machines, Panama City, Panama, 10-12 September 2018

\title{
PROPOSAL OF AIR COMPRESSING DEVICE USING WALKING VIBRATION ENERGY REGENERATION FOR PNEUMATIC DRIVEN ASSISTIVE DEVICE*
}

\author{
MANABU OKUI \\ Research and development initiative, Chuo university, 1-13-27 Kasuga \\ Bunkoy-ku, Tokyo, 112-8551, Japan \\ YASUYUKI YAMADA \\ Faculty of science and engineering, Chuo university, 1-13-27 Kasuga \\ Bunkoy-ku, Tokyo, 112-8551, Japan \\ TARO NAKAMURA \\ Faculty of science and engineering, Chuo university, 1-13-27 Kasuga \\ Bunkoy-ku, Tokyo, 112-8551, Japan
}

\begin{abstract}
Pneumatically driven wearable assistive devices for walking have been developed recently. These devices can achieve flexible assistance without control; however, they require large and heavy air compressors for activation. In this study, a pneumatically driven source using vibration energy regeneration from walking was developed. The aim was to activate the cylinder using vibrations due to walking and compressed air. A mass element, which is connected to a human body via a spring and a cylinder, vibrates along with the human gait cycle. Next, a prototype was developed and tested. In walking experiments, stored pressure was measured at several gait cycles and masses for comparison. Results indicate that the gait cycle period and masses affect the stored pressure; the highest pressure recorded was $0.08 \mathrm{MPa}$.
\end{abstract}

\section{Introduction}

Wearable assistive devices have been developed recently to prolong the health spans of the elderly or to reduce heavy manual labor [1-3]. These devices are required to provide flexible assistance in a safe and natural way. Devices driven by motors and reduction gear are controlled to be flexible by feed back control. In such cases, sensors are required and they cannot adapt to sudden external forces that are faster than the actuator's performance. In addition, such devices interfere with the wearer's movement because they have low back drivability.

\footnotetext{
${ }^{*}$ This research was supported by the Fluid Power Technology Program Foundation.
} 
In contrast, pneumatically driven assistive devices that achieve a flexible assistance without control using pneumatic artificial muscles for actuators have been developed $[4,5]$. These devices can mitigate the problems discussed earlier; however, they require large and heavy air compressors for activation. Therefore, portable pneumatically driven devices have been recently developed [6-9]. However, a mobility comparison of the gas compression methods [10] indicates that the existing methods pose certain limitations.

In this study, a novel air compression method that uses walking vibration energy regeneration is proposed. Additionally, a prototype is developed and tested.

\section{Concept and modeling}

\subsection{Vibration during walking}

The center of gravity moves vertically during walking. Fig. 1 shows the variation of height and acceleration of the center of gravity for two gait cycles. The center of gravity is low in double-stance phase and high in the single-stance phase. Acceleration is $\sim 180^{\circ}$ out of phase with height.

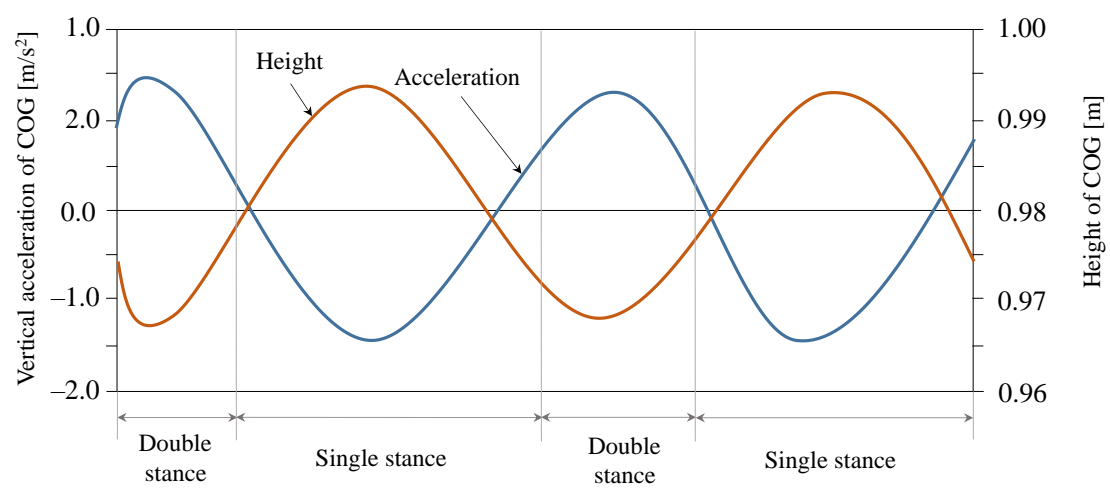

Figure 1. Height and acceleration of the center of gravity during a complete gait cycle. 


\subsection{Regeneration of walking energy}

Fig. 2 schematizes the walking energy regeneration device. The regeneration device is fixed to the wearer, and mass elements are connected via a spring and a cylinder. The movement of the mass element is restricted in the vertical direction by a slider. Due to vibrations during walking, the mass element moves up and down and drives the cylinder to compress air. This enables obtaining pneumatic energy from walking energy. The mass element comprises additional systems such as electric valves and a battery.

The inertial force generated by the vibration of the mass element is expected to assist in walking.
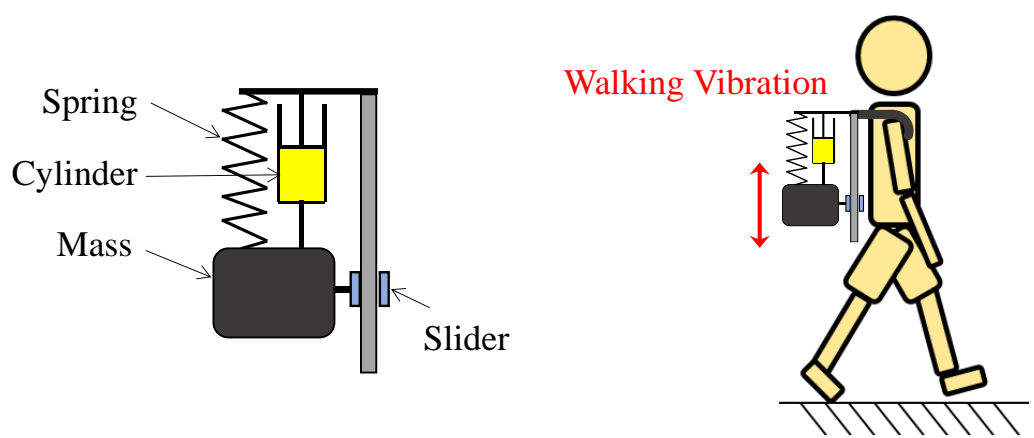

Figure 2. Operation of air compression device using walking vibration energy.

\subsection{Modeling}

The vibration phenomenon of the proposed method can be regarded as the forced displacement vibration of a spring-mass damper system, as shown in Fig. 3. Note that the mass of a human is sufficiently larger than the mass of the device. The impedance elements of the cylinder are regarded as the damper and spring elements. $k$ is the spring constant of the spring, $k_{\mathrm{c}}$ is the spring constant of the cylinder, and $d_{\mathrm{c}}$ is coefficient of viscosity of the cylinder. The resonance frequency of the system can be expressed as $(2 \pi)^{-1} \sqrt{{k m^{-1}}^{-1} 0.5 d^{2} m^{-2}}$. 


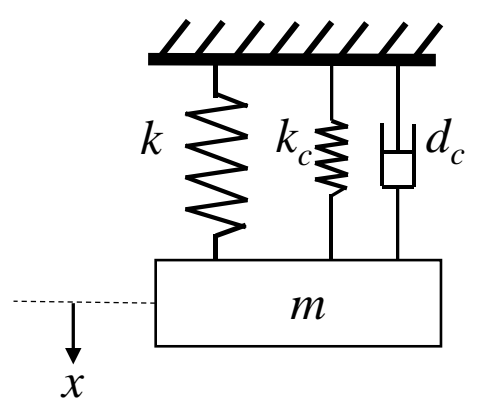

Figure 3. Model of the proposed system.

\section{Prototype}

\subsection{Design}

The design of the prototype is shown in Fig. 4. The prototype comprises a frame and a plate for loading mass elements. These two parts are connected via a spring, a cylinder for generating compressed air, and a slider that restricts movement of the plate in the vertical direction. Brackets for attaching the shoulder strap are attached to the top and bottom of the frame.

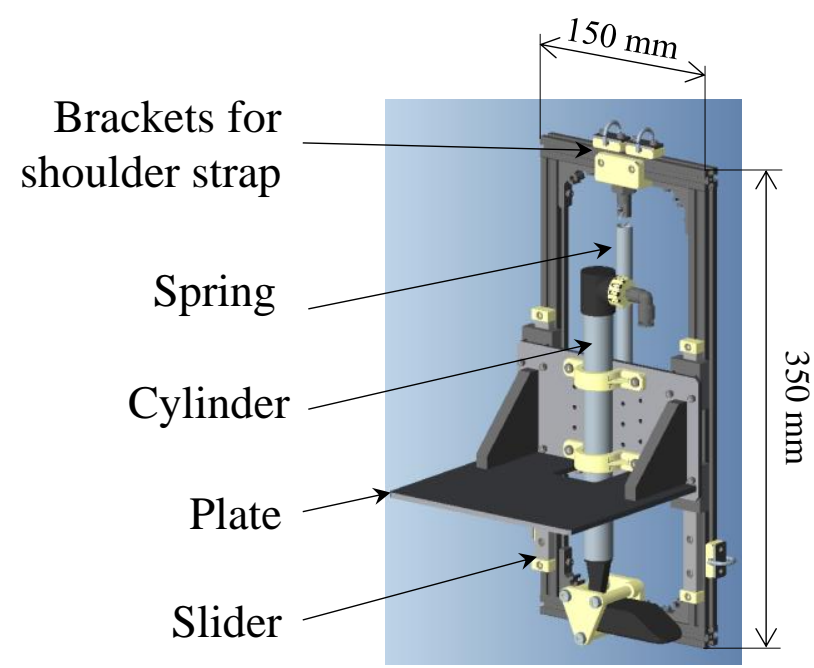

Figure 4. Design of the prototype. 


\subsection{Developed prototype}

The developed prototype is shown in Fig. 5, and the specifications are shown in Table 1 . The cylinder is composed of a commercially available double-action air pump. The discharge port of the pump is connected to the tank, which is used to store the compressed air. A check valve prevents the backflow of the compressed air, and a pressure sensor is mounted between the tank and the pump.

Table 1. Specifications of the prototype.

\begin{tabular}{cc}
\hline Weight of the prototype & $1.79 \mathrm{~kg}$ \\
\hline Weight of the plate part & $0.570 \mathrm{~kg}$ \\
\hline Size & $350 \mathrm{~mm} \times 150 \mathrm{~mm} \times 180 \mathrm{~mm}$ \\
\hline Model number of spring & $\begin{array}{c}22-1245(\text { Samini Co., Ltd.) } \\
\text { Spring constant is 0.392 N/mm }\end{array}$ \\
\hline Model number of cylinder & D59-35-301 \\
\hline Volume of tank & $600 \mathrm{ml}$ \\
\hline Length of the tube between cylinder and tank & $670 \mathrm{~mm}$ \\
\hline
\end{tabular}

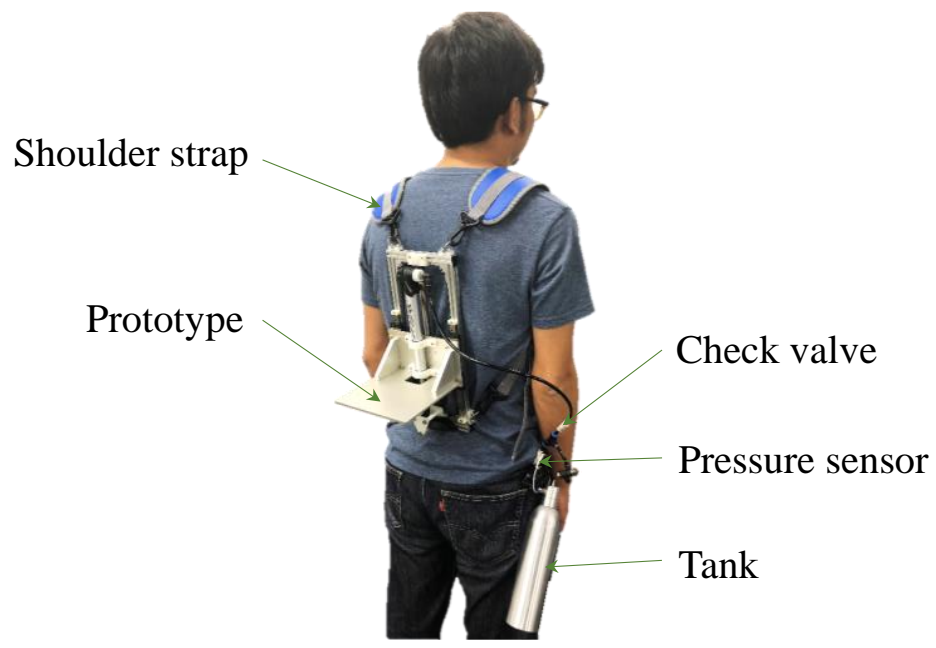

Figure 5. Subject wearing the prototype. 


\section{Experiment}

\subsection{Experimental conditions}

The prototype shown in Fig. 5 was experimentally validated. In each trial, pressure measurements were performed while the subject walked for $120 \mathrm{~s}$ with a 700-mm step length. Two parameters were varied: a) the number of weights on the plate [one to four weights $(0.85 \mathrm{~kg}$ each) were used] and b) the period of the walking cycle $(0.9,1.0$, and $1.2 \mathrm{~s})$. The subject is a male with a height of $178 \mathrm{~cm}$ and a weight of $68 \mathrm{~kg}$.

\subsection{Results and discussion}

Fig. 6 shows the experimental results, and Table 2 shows the theoretical resonance period, which was calculated assuming that $k_{\mathrm{c}}$ and $d_{\mathrm{c}}$ are 0. Fig. 6 shows the pressure profiles for three different step periods and for different weights. It is evident that short gait cycles yield more amount of compressed air. The highest pressure and fastest compressed air generation were observed for three weights and a 0.9-s gait cycle period. Here, the pressure was $\sim 0.08 \mathrm{MPa}$, sufficient for activating pneumatic actuators.

Under the two-weight condition, gait cycle periods of 1.0 and $0.9 \mathrm{~s}$ showed similar pressure profiles because this experimental condition has a $0.95-\mathrm{s}$ resonance frequency, as shown in Table 2, and is similar to the 1.0- and 0.9-s gait cycles.

However, comparing the results in Fig. 6 with the gait cycle in Table 2, the resonance frequency and experimental results did not correlate with each other were not observed. For example, in the case wherein four weights were used, the compressed air becomes largest at 1.2-s gait period. However, the experimental result showed that $0.9 \mathrm{~s}$ period provides the highest pressure.

Therefore, it is necessary to improve accuracy of the model. For example, adjusting the cylinder spring force and coefficient of viscosity according to the cylinder pressure could improve the agreement between model and experiment. Additionally, a more detailed analysis of the model could help determine the optimum values of the spring constant and weight. These proposed adjustments could improve the efficiency of this device to make it practical. 


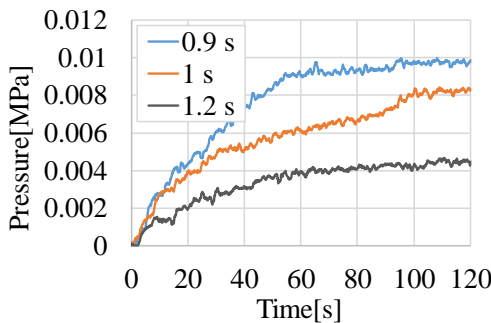

(a) one weight

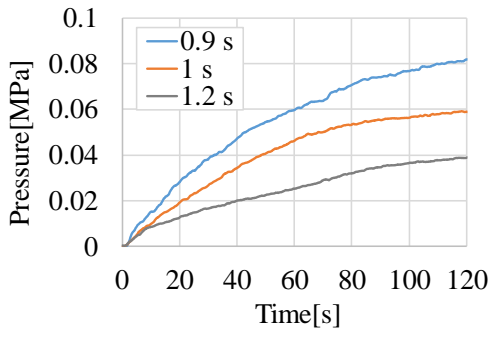

(c) three weights

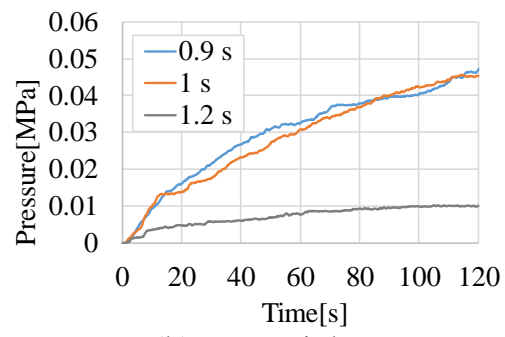

(b) two weights

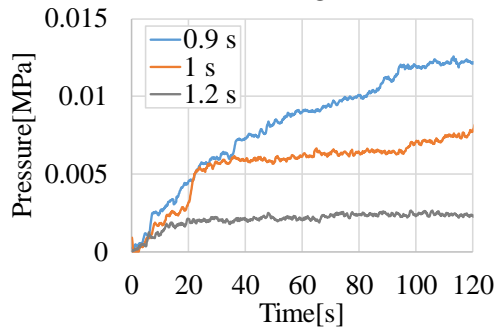

(d) four weights

Figure 6. Pressure profiles for various gait cycle periods. Each plot shows the results for a different weight.

Table 2. Resonance period of each condition

\begin{tabular}{ccc}
\hline Number of weights & Weight $[\mathrm{kg}]$ & Resonance period [s] \\
\hline \hline 1 & 1.42 & 0.38 \\
\hline 2 & 2.27 & 0.48 \\
\hline 3 & 3.12 & 0.56 \\
\hline 4 & 3.97 & 0.63
\end{tabular}

\section{Conclusion}

In this study, an air pressure source using regeneration of energy from walking was proposed and a prototype was constructed and tested. In the walking experiments, pressure profiles during gait cycles were compared at varying gait cycle periods and masses. The highest pressure in the tank was $0.08 \mathrm{MPa}$ for a weight of $3.12 \mathrm{~kg}$ and gait cycle period of $0.9 \mathrm{~s}$. Plans for future study include the optimization of the device specifications using a more detailed model.

\section{Acknowledgments}

This research was supported by the Fluid Power Technology Program Foundation. 


\section{References}

1. T. Hayashi, H. Kawamoto and Y. Sankai, "Control Method of Robot Suit HAL working as Operator's Muscle using Biological and Dynamical Information," In Proc. IEEE/RSJ Int. Conf. on Intelligent Robots and Systems (IROS), pp.3063-3068. 2005.

2. Y. Lee, S. Roh, M. Lee, B. Choi, J. Lee, J. Kim, H. Choi, Y. Shim and Y. Kim, "A Flexible Exoskeleton for Hip Assistance" In Proc. IEEE/RSJ Int. Conf. on Intelligent Robots and Systems (IROS), pp.1058-1063, 2017.

3. A. Tsukahara and M. Hashimoto, "Pilot Study of Single-Legged Walking Support using Wearable Robot based on Synchronization Control for Stroke Patients", Proceedings of the 2016 IEEE International Conference on Robotics and Biomimetics, pp.886-891, Qingdao, China, December 3-7, 2016.

4. A. Ohno, H. Nabae and K. Suzumori, "Static Analysis of Powered LowBack Orthosis driven by thin pneumatic artificial muscles considering body surface deformation", In Proc. of 2015 IEEE/SICE International Symposium on System Integration (SII), pp.39-44, 2015.

5. M. Okui, S.Iikawa, Y.Yamada and T. Nakamura, "Variable viscoelastic joint system and its application to exoskeleton", Proc. of The 2017 IEEE/RSJ International Conference on Intelligent Robots and Systems(IROS), Vancouver, Canada, pp.3897-3902, 2017.

6. M. Okui, S.Iikawa, Y.Yamada and T. Nakamura, "A pneumatic power source using a sodium bicarbonate and citric acid reaction of for use in mobile devices", Proc. of The 2017 IEEE/RSJ International Conference on Intelligent Robots and Systems(IROS), Vancouver, Canada, pp.1040-1045, 2017.

7. K. Suzumori, A. Wada and S. Wakimoto, "A new mobile pressure control system for pneumatic actuators using reversible chemical reactions of water, IEEE/ASME International Conference on Advanced Intelligent Mechatronics(AIM), pp.122-127, 2013.

8. K.Nakahara, K.Narumi,R.Niiyama, Y.Kawahara, "Electric Phase-change Actuator with Inkjet Printed Flexible Circuit for Printable and Integrated Robot Prototyping", IEEE International Conference on Robotics and Automation (ICRA), pp.1856-1863, 2017.

9. M. Wehner, M. T. Tolley, Y. Menguc, Y. Park, A. Mozeika, Y. Ding, C. Onal, R. F. Sheperd, G. M. Whitesides, R. J. Wood, "Pneumatic Energy Sources for Autonomous and Wearable Soft Robotics", Soft robotics, vol.2, No.00. pp. 1-12, 2012.

10. M. Okui, Y. Nagura, Y. Yamada, T. Nakamura, "Hybrid Pneumatic Source Based on Evaluation of Air Compression Methods for Portability", IEEE Robotics and Automation Letters. vol. 3-2, pp. 819-826, 2018 